\title{
EXTRACELLULAR DIFFERENTIAL PROTEOME ANALYSIS OF SUBSTRATES OF DIFFERENT LIGNIN MODEL COMPOUNDS DEGRADED BY ASPERGILLUS FUMIGATUS G-13
}

\author{
Jinda $\mathrm{LI}^{1,2}$, Xia DU ${ }^{1,2}$, Qian $\mathrm{FENG}^{1,2}$, Hong YAN 1 1, $2^{*}$ \\ ${ }^{1}$ College of Chemical and Environmental Engineering, Harbin University of Science and Technology, \\ Harbin 150040, China \\ ${ }^{2}$ Key Laboratory of Green Chemical Technology of College of Heilongjiang Province, Harbin 150040, China
}

Received 23 September 2019; accepted 23 March 2020

\begin{abstract}
Highlights:
The iTRAQ proteomics technique was used for the first time to analyze the extracellular secreted protein of the lignin model compound fermented by Aspergillus fumigatus G-13.

$>$ Different lignin model compounds have significant effects on bacterial metabolism and extracellular secreted proteins.

The key enzyme involved in the degradation of lignin model compounds is ferulic acid esterase, and the energy metabolism pathway plays an important auxiliary role.
\end{abstract}

\begin{abstract}
Abstact. Aspergillus fumigatus G-13 has the potential to degrade lignocellulose biomass. The purpose of this work is to analyze the extracellular soluble secretory protein of lignocellulose degradation by Aspergillus fumigatus G-13. The research used ferulic acid, sinapic acid and p-coumaric acid as carbon sources. By controlling the culture conditions, adding cellulose co-substrate and auxiliary carbon source, the enzymatic production law of Aspergillus fumigatus G-13 degradation of three lignin model compounds was investigated. The two groups with the greatest difference in enzyme activity expression were screened, and high throughput quantitative proteomics analysis using iTRAQ. iTRAQ analysis showed that a total of 3862 protein spots changed significantly, of which 2103 down-regulated proteins and 1759 up-regulated proteins. The differential proteins involved in the degradation process of lignin model compounds are concentrated in dioxygenase, oxidoreductase, ferulic acid esterase B-2, isoamyl alcohol oxidase, bifunctional catalase peroxidase CAT2, cellulase, cytochrome P450 monooxygenase, flavin-binding monooxygenase, etc. Lignin-related differential abundance proteins were mapped to 128 metabolic pathways. Significantly enriched pathways include metabolic pathways, glyceride metabolism, oxidative phosphorylation, riboflavin metabolism, peroxisomes, riboflavin metabolism. The information presented in this paper is helpful to better understand the lignocellulose degradation mechanisms of A. fumigatus G-13.
\end{abstract}

Keywords: Aspergillus fumigatus G-13, biodegradation, lignin model compound, iTRAQ, lignin degradation mechanism.

Online supplementary material: Supporting information for this paper is available as online supplementary material at https://doi.org/10.3846/jeelm.2020.12695

\section{Introduction}

Lignin is an amorphous aromatic polymer widely presented in plants, which contained a structural unit of oxyphenylene alcohol or a derivative thereof in its molecular structure (Tao \& Guan, 2003). At present, most lignin is directly used as fuel or discharged into rivers, which not only wastes resources but also causes environmental pollution (Himmel et al., 2007). The biodegradation of lignin has been a worldwide research focus and difficult problem.
Clarifying the biodegradation mechanism of lignin is not only the primary key scientific issue which needs to be elucidated, but also the urgent theoretical basis for solving the practical application problems. The study of lignin degradation mechanism needs to follow the change of substrate and start from enzyme itself. It's a lot of work to figure out these problems, and some trace proteins are easily overlooked. Proteomics is an effective means to explore protein action patterns, functional mechanisms, and regulation control as well as interrelationship within the

*Corresponding author. E-mail: yanhong204821@aliyun.com 
protein population at an overall level. It has a significant role in revealing the basic laws of microbial degradation of lignin (Grabber, 2005; Wilkins et al., 1996).

At present, researchers have used proteomics technology to find and identify some enzymes that play a role and their metabolic pathways in the degradation of lignin. Adav et al. (2015) used iTRAQ technology to study the proteome of Aspergillus fumigatus and found that cellulose can stimulate the production of important lignocellulosic hydrolase. Ji et al. (2012) first used the Shotgun proteomics strategy to analyze the secretory proteome of Trametes trogii WT-1. A total of 64 proteins including lipid metabolism proteins, carbohydrate metabolismrelated proteins, protein metabolism-related proteins, cell walls and lignin degradation-related proteins were identified. Ray et al. (2012) used liquid chromatography-tandem mass spectrometry to perform proteomics studies on extracellular fluids. The study identified 57, 116 and 102 degrading enzymes respectively. MacDonald et al. (2012) studied the effect of lignin conditions on expression of microsomal proteins in Phanerochaete chrysosporium RP-78 strain, and found that transporters and cytochrome P450s were involved in the degradation process of lignin and played an important role. Dawoud and Abu-Taleb (2012), through the study of intracellular proteomics of lignocellulose-degrading bacteria, a large number of information related to its metabolic regulation and stress-related expression proteins can be obtained. Arulmani et al. (2011) analyzed the lignin degradation model organism P. chrysosporium secreted proteome, iTRAQ data indicated that endoglucanase, exoglucanase, 3-glucosidase, and cellobiose dehydrogenase gene expression were significantly up-regulated under cellulose and cellulose lignin culture conditions. These studies provide us with a feasible reference to explain the degradation mechanism of lignin-degrading enzyme by using proteomics technology.

At present, there is no research on the mechanism of proteomics technology for the degradation of lignin by Aspergillus fumigatus. Therefore, the iTRAQ proteomics technique was used for the first time to analyze the extracellular secreted protein of the lignin model compound fermented by Aspergillus fumigatus G-13. The key enzymes of Aspergillus fumigatus G-13 degradation of the lignin model compounds and their regulatory mechanisms were clarified, and the complex and continuous collaborative changes of metabolic regulation pathway in the process of lignin degradation were described. Finally, identify the key enzymes and important auxiliary pathways.

As a common lignin monomer model compound, pcoumaric acid and sinapic acid are direct precursors in the process of lignin biosynthesis, and represent the structure of lignin better than other model compounds, they are widely used to study lignin biodegradation. Therefore, this study intends to use the lignin monomer model compound as a substrate, using the strain Aspergillus fumigatus G-13 for fermentation. Then, the proteomic analysis of the sample is performed using iTRAQ technology, and the enzyme composition and classification of the lignindegrading enzyme involved in the strain are obtained. In this way, the efficient secretion expression and mechanism of the lignin structural monomer model compound exogenous protein were investigated. It layed a foundation for the study of the production of strain lignin degrading enzyme and the mechanism of biodegradation of lignin.

\section{Materials and methods}

\subsection{Strain}

Aspergillus fumigatus (A. fumigatus) G-13, which could degrade lignin used in this work was isolated from the samples collected from soil near the sewage draining exit of a paper mill in Harbin.

\subsection{Instruments}

Shimadzu LC-20AB liquid chromatograph, Shimadzu LC20AD nanoliter liquid chromatograph, ESC tandem mass spectrometer: Triple TOF 5600 (SCIEX, Framingham, MA, USA), Ion source is Nanospray III source (SCIEX, Framingham, MA, USA), Emitter is a needle drawn from quartz material (New Objectives, Woburn, MA, USA).

\subsection{Preparation of bacterial suspension}

The oblique surface of $A$. fumigatus G-13 PDA medium stored at $4{ }^{\circ} \mathrm{C}$ was taken out, and the fungal spores were washed with sterile water to prepare a spore suspension of $10^{6}$ cells $/ \mathrm{mL}$ (measured by blood cell counting method). Keep it in a $4{ }^{\circ} \mathrm{C}$ refrigerator.

\subsection{Fermentation culture}

Using a liquid culture method, added $100 \mathrm{~mL}$ of a large amount of elemental nutrient solution and $0.1 \mathrm{~mL}$ of a trace element nutrient solution to a $250 \mathrm{~mL}$ Erlenmeyer flask, wherein the concentration of lignin monomer model compound was $0.1 \mathrm{mmol} / \mathrm{L}$, cellulose co-substrate and glucose concentration were both $10 \mathrm{~g} / \mathrm{L}$, autoclave at $120^{\circ} \mathrm{C}$ for $20 \mathrm{~min}$. Then, added $4 \mathrm{~mL}$ of the spore suspension and cultured at a temperature of $30^{\circ} \mathrm{C}$. When using shake flask culture, the rotation speed was $160 \mathrm{r} / \mathrm{min}$. Samples were taken at the 3rd, 6th, 9th, 12th, and 15th day of culture, and measured the enzyme activity. Wherein each set of three replicates. The two groups with the largest difference in enzyme activity were used as samples for subsequent proteomic analysis. The substrate composition and culture method corresponding to the sample number are shown in Table S1.

\section{5. iTRAQ analysis of protein samples}

\subsubsection{Sample concentrate}

Took fermentation broth to a $30 \mathrm{~K}$ ultrafiltration tube and centrifuged at $6000 \mathrm{r} / \mathrm{min}$ for $15 \mathrm{~min}$. Repeated this step several times until the culture medium was separated from mycelium, and the substance intercepted by ultrafiltration membrane was a concentrated protein sample. 


\subsubsection{Extraction of protein}

Weigh the appropriate amount of sample, added Lysis Buffer, added PMSF with a final concentration of $1 \mathrm{mmol} / \mathrm{L}$ and EDTA at $2 \mathrm{mmol} / \mathrm{L}$, allowed to stand for $5 \mathrm{~min}$ after vortexing oscillation, then added DTT with a final concentration of $10 \mathrm{mmol} / \mathrm{L}$, ice bath ultrasounded $5 \mathrm{~min}$, and the supernatant was extracted after centrifugation for $20 \mathrm{~min}$. In water bath at $56^{\circ} \mathrm{C}$, added $10 \mathrm{mmol} / \mathrm{L}$ DTT to the supernatant for $1 \mathrm{~h}, 55 \mathrm{mmol} / \mathrm{L}$ IAM was added to the supernatant after restoring to room temperature, and then placed in darkroom for $45 \mathrm{~min}$. Added 5 volumes of pre-cooled acetone to the supernatant again, precipitated at $-20{ }^{\circ} \mathrm{C}$ for $2 \mathrm{~h}$, centrifuged at $4{ }^{\circ} \mathrm{C}$ for $20 \mathrm{~min}$, discarded the supernatant. This step was repeated 2-3 times until the supernatant was colorless. Finally, an appropriate amount of Lysis Buffer 3 was added to the precipitate for redissolution, after $5 \mathrm{~min}$ of ultrasound in ice bath, it was centrifuged at $4{ }^{\circ} \mathrm{C}$ for $20 \mathrm{~min}$ at a speed of $25000 \mathrm{r} / \mathrm{min}$, and the supernatant was taken for quantification. Protein concentration was determined according to Bradford quantitative method and the amount of protein required for SDS-PAGE and iTRAQ analysis was determined.

\subsubsection{Protein extraction quality control}

Bradford quantification: standard protein $(0.2 \mu \mathrm{g} / \mu \mathrm{L}$ BSA $)$ $0,2,4,6,8,10,12,14,16$ and $18 \mu \mathrm{L}$ were added to the 96 well ELISA plate A1 to A10, respectively. Then, 20, 18, 16, $14,12,10,8,6,4$, and $2 \mu \mathrm{L}$ of pure water were sequentially added, and then $180 \mu \mathrm{L}$ of Coomassie Brilliant Blue G-250 quantitative working solution was added to each well. The protein to be tested was diluted several times, and $180 \mu \mathrm{L}$ of the quantitative working solution was added to $20 \mu \mathrm{L}$ of the protein solution to read $\mathrm{OD}_{595}$. The sample protein concentration was calculated based on the standard curve and sample $\mathrm{OD}_{595}$.

\subsubsection{Proteolysis}

$100 \mu \mathrm{g}$ of protein solution was taken from each sample, $2.5 \mu \mathrm{g}$ of Trypsin enzyme was added in a ratio of protein: enzyme $=40: 1$, and enzymatic hydrolysis at $37^{\circ} \mathrm{C}$ for $4 \mathrm{~h}$. After that, Trypsin was added in the above ratio, and the enzymatic hydrolysis time was increased to $8 \mathrm{~h}$. After enzymatic hydrolysis, the peptide segment was desalted by Strata X column.

\subsubsection{Separation of peptide segment markers}

After the reagents were returned to room temperature, added $50 \mu \mathrm{L}$ of isopropanol to each tube of reagent. After vortexing, centrifuged at low speed and took out the iTRAQ label reagent according to number of samples. The peptide segment sample was dissolved in $0.5 \mathrm{~mol} / \mathrm{L}$ TEAB and added to the corresponding iTRAQ labeling reagent, allowed to stand at room temperature for $2 \mathrm{~h}$. The samples were separated using a Shimadzu LC-20AB liquid phase system (Separation column was $5 \mu \mathrm{m} 4.6 \times 250 \mathrm{mmol} / \mathrm{L}$ Gemini $\mathrm{C}_{18}$ column).

\subsubsection{High performance liquid chromatography}

The lyophilized peptide sample was redissolved with mobile phase A ( $2 \% \mathrm{ACN}, 0.1 \% \mathrm{FA})$, centrifuged at $20000 \mathrm{r} / \mathrm{min}$ for $10 \mathrm{~min}$, and the supernatant was taken for sampling. Separation was performed by Shimadzu LC20AD model liquid chromatograph.

\subsubsection{Mass spectrometry}

The peptide segment separated by liquid phase enters the ESI tandem mass spectrometer, the parameters were set as follows: ion source spray voltage $2300 \mathrm{~V}$, nitrogen pressure $30 \mathrm{psi}$, spray pressure $15 \mathrm{psi}$, spray interface temperature $150{ }^{\circ} \mathrm{C}$. Scanning in high sensitivity mode, the first mass spectrometry scan has a cumulative time of $250 \mathrm{~ms}$ and the scan quality ranges from 350 to $1500 \mathrm{Da}$. Based on the first-level scan information, according to the intensity in the first-order spectrogram from high to low, the first 30 first-order spectra with intensity exceeding $150 \mathrm{cp}$ were selected for fragmentation, and scanned the secondary information. The screening criteria were as follows: $\mathrm{m} / \mathrm{z}$ range was 350-1250 Da; the number of electric charges was 2-5. The parent ion dynamic exclusion is set to: within half of the peak time (about $12 \mathrm{~s}$ ), the fragmentation of the same parent ion was not more than 2 times. The scan time for the secondary mass spectrum was $100 \mathrm{~ms}$. For iTRAQ-type data acquisition, the fragmentation energy selection was adjusted according to the iTRAQ reagent, and the first quadrupole Q2 has an off-transmission efficiency of $100 \%$ at $100 \mathrm{Da}$.

\subsubsection{Biological information analysis}

After the original mass spectrometry data were converted into mgf format files, protein identification software Mascot was used to search and identify the corresponding database. At the same time of quality control analysis, judged whether the data was qualified. After the data was qualified, a certain screening threshold, the final credible protein identification result was obtained. Subsequently, iTRAQ quantitative analysis was carried out, and significant difference proteins were screened from the quantitative results. Finally, GO, Pathway, COG and other functional annotations and GO and Pathway enrichment analysis of differential proteins were performed.

\section{Results}

\subsection{Analysis of enzyme activity of $A$. fumigatus G-13 degrading lignin model compounds}

Figure 1 is the time-dependent curves of the enzyme activities of lignin peroxidase (Lip), laccase (Lac) and manganese peroxidase (Mnp) produced by ferulic acid, p-coumaric acid and sinapic acid as fermentation substrates, respectively. It can be seen that within 15 days of fermentation culture, the activities of three ligninases produced by $A$. fumigatus G-13 were all increased first, then decreased, and eventually tended to be gentle, but the highest enzyme activity appeared at different time. 

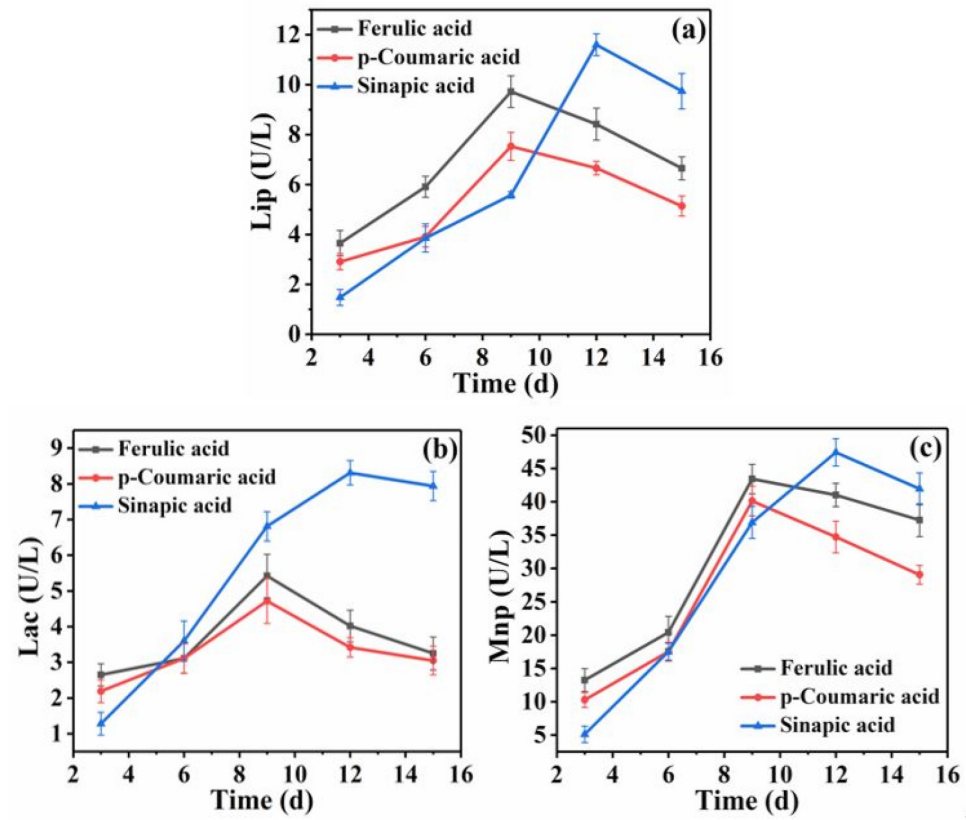

Figure 1. The change of enzyme activity in lignin model compounds fermented by A. fumigatus:

(a) - Lignin peroxidase; (b) - Laccase; (c) - Manganese peroxidase

The three enzyme activities of ferulic acid and p-coumaric acid as substrate showed the highest value on the 9th day, the Lip, Lac, and Mnp activities were $9.65 \mathrm{U} / \mathrm{L}, 5.63 \mathrm{U} / \mathrm{L}$, $43.35 \mathrm{U} / \mathrm{L}$ and $7.52 \mathrm{U} / \mathrm{L}, 4.84 \mathrm{U} / \mathrm{L}, 40.36 \mathrm{U} / \mathrm{L}$, respectively. While the highest enzyme activity with sinapic acid as a substrate appeared at $12 \mathrm{~d}$. However, the highest values of

Table 1. Effects of adding other substrates or changing culture methods on various ligninase activities produced by $A$. fumigatus $\mathrm{G}-13$

\begin{tabular}{|c|c|c|c|c|c|c|c|}
\hline $\begin{array}{c}\text { Num- } \\
\text { ber }\end{array}$ & LiP & Lac & MnP & $\begin{array}{c}\text { Num- } \\
\text { ber }\end{array}$ & LiP & Lac & MnP \\
\hline A & 100 & 100 & 100 & A-6 & 206 & 256 & 193 \\
\hline B & 100 & 100 & 100 & B-6 & 198 & 235 & 220 \\
\hline C & 100 & 100 & 100 & C-6 & 209 & 254 & 235 \\
\hline A-1 & 123 & 176 & 160 & A-7 & 246 & 292 & 263 \\
\hline B-1 & 92 & 120 & 87 & B-7 & 215 & 225 & 254 \\
\hline C-1 & 185 & 220 & 190 & C-7 & 274 & 360 & 297 \\
\hline A-2 & 250 & 268 & 286 & A-8 & 375 & 398 & 321 \\
\hline B-2 & 288 & 293 & 302 & B-8 & 397 & 405 & 391 \\
\hline C-2 & 271 & 364 & 387 & C-8 & 330 & 394 & 428 \\
\hline A-3 & 345 & 340 & 413 & A-9 & 457 & 402 & 493 \\
\hline B-3 & 421 & 369 & 465 & B-9 & 486 & 541 & 593 \\
\hline C-3 & 522 & 475 & 452 & C-9 & 587 & 529 & 641 \\
\hline A-4 & 377 & 460 & 395 & A-10 & 754 & 898 & 804 \\
\hline B-4 & 320 & 398 & 341 & B-10 & 819 & 865 & 914 \\
\hline C-4 & 412 & 568 & 563 & C-10 & 1032 & 1250 & 1096 \\
\hline A-5 & 463 & 526 & 322 & A-11 & 901 & 965 & 997 \\
\hline B-5 & 388 & 465 & 313 & B-11 & 1024 & 1132 & 1089 \\
\hline C-5 & 494 & 608 & 588 & C-11 & 1156 & 1285 & 1164 \\
\hline
\end{tabular}

three ligninase activities produced by fermentation with sinapinic acid were higher than those of the other two substrates, and Lip, Lac and Mnp enzyme activities were $11.52 \mathrm{U} / \mathrm{L}, 8.53 \mathrm{U} / \mathrm{L}$ and $47.14 \mathrm{U} / \mathrm{L}$, respectively. It can be seen that sinapic acid as substrate is more favorable for the strain to produce higher ligninase activity, but the time required for enzyme to reach the peak is slightly longer. This trend is basically the same as that reported by Li et al. on the degradation of lignin model compounds by bacteria, that is, sinapic acid is utilized by the strain later than ferulic acid or p-coumaric acid (Li et al., 1999).

By controlling the addition of cellulose co-substrate or glucose and changing the way of culture method to investigate the effect of strain produce lignin degrading enzyme activity. Under various control conditions, the highest value of various ligninase activities produced by fermentation of $A$. fumigatus G-13 within 15 days was used as an evaluation index. Table 1 shows the effect of adding other substrates or changing culture method on the activity of lignin-degrading enzyme produced by $A$. fumigatus G-13. Among them, each data was obtained by using the corresponding lignin monomer model compound as substrate, the enzyme activity of all kinds of lignin produced was defined as $100 \%$, thus obtaining the relative enzyme activity.

According to the results in Table 1, when added glucose as an auxiliary carbon source and added cellulose model compound under the condition of shaking flask culture, the activity of $A$. fumigatus G-13 lignin-degrading enzyme with sinapic acid as substrate changed the most. The relative enzyme activities of Lip, Lac and Mnp were increased by $1156 \%, 1285 \%$ and $1164 \%$, respectively. Under static culture conditions, the addition of microcrystalline cellulose also promoted fermentation of 
ferulic acid and sinapic acid substrates by A. fumigatus G-13. For the liquid fermentation of $A$. fumigatus with $\mathrm{p}-$ coumaric acid as substrate, the relative enzyme activities of $\mathrm{LiP}, \mathrm{Lac}$ and $\mathrm{MnP}$ increased to $92 \%, 120 \%$ and $87 \%$, respectively. A. fumigatus $\mathrm{G}-13$ is an aerobic fungus, and the oscillatory method helps the circulation of oxygen. Therefore, it was beneficial to the growth of strain and produced more secreted proteins than static culture. Therefore, the p-coumaric acid and microcrystalline cellulose were used as carbon source (ie B-1) under static culture conditions and sinapic acid, carboxymethyl cellulose and glucose were used as carbon sources (ie C-11) under shake flask culture conditions were selected as samples for further proteomic analysis.

\subsection{Protein quantification}

Extraction of protein samples by acetone precipitation, and the protein of A. fumigatus G-13 cells under different substrate conditions for growth on day 9 was quantified by the Bradford method. Two groups of samples B-1 and C-11 were tested in three parallel ways, numbered b-1, b-2, b-3 and $c-1, c-2$ and $c-3$, respectively. The quantitative results are shown in Table S2. The protein quantification results were consistent with the results of previous experimental lignin degrading enzyme activity, and the total protein content of B-1 was significantly lower than that of C-11.

\subsection{Protein SDS-PAGE results}

The protein sample was subjected to SDS-PAGE electrophoresis, and the gel electrophoresis pattern is shown in Figure S1. Among them, strips 1, 2 and 3 represented B-1 samples, and strips 4, 5 and 6 represented C-11 samples. It can be seen that the bands of 6 samples are clear, there are no miscellaneous bands, and there is no protein degradation phenomenon, the strips and repeatability are qualified, and each strip is relatively parallel, the total protein satisfies $2-3$ analysis conditions, subsequent iTRAQ analysis can be performed.

\subsection{Mass spectrometric identification of protein samples}

\subsubsection{Differential protein point analysis}

The three parallels of two groups of A. fumigatus G-13 were b-1, b-2, b-3 and c-1, c-2, c-3, and a total of 343,789 spectrogram were generated. Under the " $1 \%$ FDR" filtration standard, a total of 29,138 peptide fragments and 4,963 proteins were identified. Table S3 shows the results of protein identification.

Figure S2 and Figure S3 show the analysis of overall protein data after three replicates of two samples. Figure S2 shows the mass distribution of the identified proteins. It can be seen that most of the proteins identified by iTRAQ technology have molecular weight of $30-70 \mathrm{kDa}$, and more than $12 \%$ of the proteins with molecular weight greater than $100 \mathrm{kDa}$ have been identified. Figure S3 shows the length distribution of the identified peptide segments. It can be seen that the number of amino acids contained in the peptide segment is mainly between 5 and 20, and the number of amino acids with the most frequent occurrence of peptide segment is mainly between 7 and 15 .

\subsubsection{Differential protein point analysis within the group}

The quantitative process of iTRAQ was realized by software IQuant. In this study, b-1 VS c-1, b-1 VS c-2, b-1 VS c-3, b-2 VS c-1, b-2 VS c-2, b-2 VS c-3, b-3 VS c-1, b-3 VS c-2, b-3 VS c-3, B-1 VS C-11, b-2 VS b-1, b-3 VS b-1,

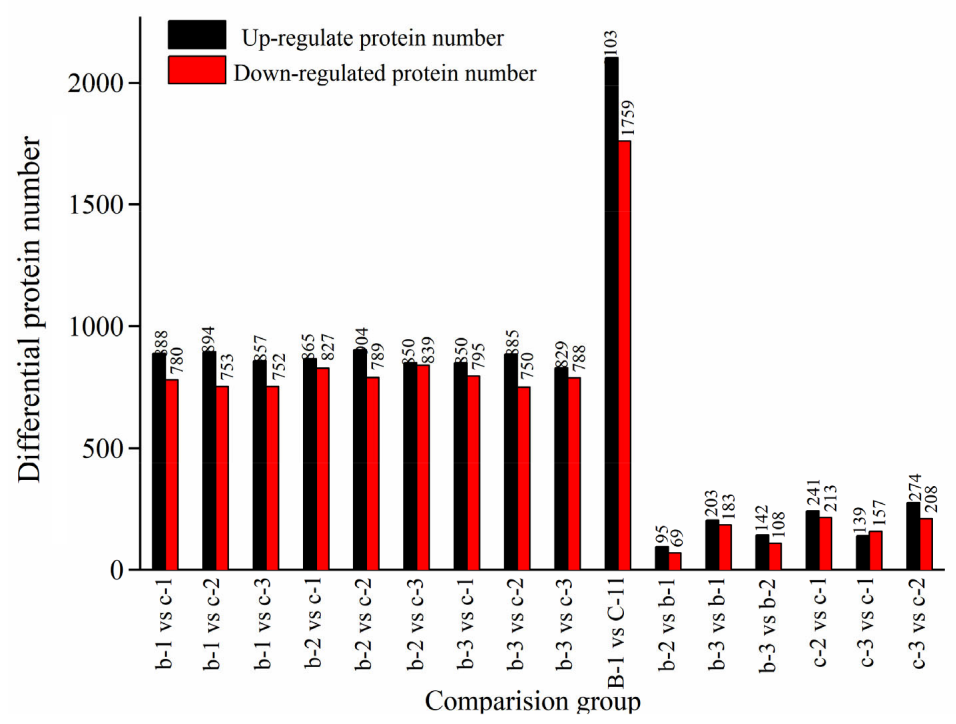

Figure 2. Bar chart of differential protein:

b: p-coumaric acid was used as a substrate, microcrystalline cellulose was added,

nd static culture; c: sinapic acid was used as a substrate, carboxymethyl cellulose and glucose were added, shake-flask culture. B-1 and C-11 were the sum of significantly changed proteins found in the experimental group 
Table 2. Lignin degradation related differential protein information

\begin{tabular}{|c|c|c|c|}
\hline Protein_ID & Description & B-1:C-11 & NCBInr Accession \\
\hline EAL84693.1 & indoleamine 2,3-dioxygenase family protein & 0.218 & gi|70982406|ref|XP_746731.1| \\
\hline EAL85498.1 & dioxygenase & 0.161 & gi|70984022|ref|XP_747536.1| \\
\hline KMK60141.1 & flavin containing amine oxidase, putative & 0.165 & gi|846914323|gb|KMK60141.1| \\
\hline KEY82638.1 & glyoxylase & 0.237 & gi|666435170|gb|KEY82638.1| \\
\hline KMK55985.1 & oxidoreductase & 0.275 & gi|846910058|gb|KMK55985.1| \\
\hline EDP50447.1 & fatty acid oxygenase PPOA & 1.294 & gi|70993806|ref|XP_751750.1| \\
\hline EAL87300.1 & fructosyl amine:oxygen oxidoreductase & 0.054 & gi|70988976|ref|XP_749338.1| \\
\hline KMK58337.1 & isoamyl alcohol oxidase, putative & 0.015 & gi|846912469|gb|KMK58337.1| \\
\hline EAL85874.2 & peroxisomal membrane protein PMP47 & 0.17 & gi|146324046|ref|XP_747912.2| \\
\hline KMK58631.1 & mycelial catalase CAT1 & 0.507 & gi|846912779|gb|KMK58631.1| \\
\hline KMK60063.1 & cytochrome P450 monooxygenase GLIC2 & 0.227 & gi|846914245|gb|KMK60063.1| \\
\hline KEY82116.1 & peroxisomal 3 ketoacyl coA thiolase KAT1 & 0.253 & gi|666434615|gb|KEY82116.1| \\
\hline EDP49472.1 & ferulic acid esterase B-2 & 0.234 & gi|298351669|sp|B0Y7U1.1|FAEB2_ASPFC \\
\hline EAL91965.1 & peroxisomal membrane protein (PEX3) & 0.017 & gi|70998562|ref|XP_754003.1| \\
\hline EDP55336.1 & monooxygenase, putative & 0.015 & gi|159130223|gb|EDP55336.1| \\
\hline KEY79055.1 & bifunCTional catalase peroxidase CAT2 & 0.601 & gi|666431450|gb|KEY79055.1| \\
\hline EAL84220.1 & ferulic acid esterase B-1 & 0.227 & gi|70981456|ref|XP_731510.1| \\
\hline KEY83653.1 & cytochrome P450 monooxygenase & 0.11 & gi|666436291|gb|KEY83653.1| \\
\hline KMK57185.1 & hypothetical protein Y699_06726 & 0.086 & gi|846911295|gb|KMK57185.1| \\
\hline EDP50869.1 & cytochrome P450 monooxygenase, putative & 0.028 & gi|159125752|gb|EDP50869.1| \\
\hline KMK56018.1 & amine oxidase & 0.109 & gi|846910091|gb|KMK56018.1| \\
\hline KMK62928.1 & cellulase, putative & 0.313 & gi|846917158|gb|KMK62928.1| \\
\hline EAL90166.1 & flavin-binding monooxygenase & 0.026 & gi|70994854|ref|XP_752204.1| \\
\hline EAL88562.1 & $\beta$-glucancellobiohydrolase & 0.137 & gi|70991503|ref|XP_750600.1| \\
\hline KEY75915.1 & homogentisate 1,2 dioxygenase & 0.191 & gi|666428261|gb|KEY75915.1| \\
\hline KEY82239.1 & cellobiose dehydrogenase & 0.02 & gi|666434745|gb|KEY82239.1| \\
\hline KEY78684.1 & peroxidase, putative & 1.385 & gi|666431072|gb|KEY78684.1| \\
\hline EAL91860.1 & peroxisomal copper amine oxidase & 0.066 & gi|70998352|ref|XP_753898.1| \\
\hline KMK60608.1 & isoamyl alcohol oxidase & 0.42 & gi|846914790|gb|KMK60608.1| \\
\hline EAL89751.1 & 3-demethyl quinone-9-methyltransferase & 1.3 & gi|70993884|ref|XP_751789.1| \\
\hline KMK57019.1 & O-methyltransferase, putative & 1.83 & gi|846911120|gb|KMK57019.1| \\
\hline EAL86791.1 & galactose oxidase & 3.52 & |gi|70986678|ref|XP_748829.1| \\
\hline EAL88473.1 & ketoreductase & 1.86 & gi|70991324|ref|XP_750511.1| \\
\hline EAL86587.1 & catechol dioxygenase & 5.89 & gi|70986262|ref|XP_748625.1| \\
\hline KEY84070.1 & aldo keto reductase AKR & 1.34 & gi|666436853|gb|KEY84070.1| \\
\hline EAL92901.1 & pyridoxamine phosphate oxidase & 2.88 & gi|71000511|ref|XP_754939.1| \\
\hline EAL85120.1 & $\begin{array}{l}\text { fumagillin biosynthesis antibiotic biosynthesis } \\
\text { monooxygenase superfamily monooxygenase }\end{array}$ & 1.23 & gi|70983261|ref|XP_747158.1| \\
\hline KEY78179.1 & fatty acid oxygenase PPOC, partial & 1.46 & gi|666430557|gb|KEY78179.1| \\
\hline EAL93430.1 & peroxisomal 3-ketoacyl-CoA thiolase (KAT1) & 2.25 & gi|71001574|ref|XP_755468.1| \\
\hline EAL89119.1 & NADH-ubiquinone oxidoreductase $39 \mathrm{kDa}$ subunit & 1.39 & gi|70992617|ref|XP_751157.1| \\
\hline KMK57661.1 & catechol dioxygenase & 2.52 & gi|846911793|gb|KMK57661.1| \\
\hline EAL88695.1 & protoporphyrinogen oxidase & 2.06 & gi|70991769|ref|XP_750733.1| \\
\hline KMK59768.1 & quinone oxidoreductase & 1.48 & gi|846913940|gb|KMK59768.1| \\
\hline EDP53821.1 & FAD monooxygenase, putative & 1.57 & gi|159128707|gb|EDP53821.1| \\
\hline KEY82179.1 & quinone oxidoreductase & 3.53 & gi|666434681|gb|KEY82179.1| \\
\hline KEY83800.1 & phytanoyl-CoA dioxygenase family protein & 1.95 & gi|666436470|gb|KEY83800.1| \\
\hline EAL93849.1 & FAD monooxygenase & 2.69 & gi|71002412|ref|XP_755887.1| \\
\hline EAL86869.1 & a-ketoglutarate-dependent taurine dioxygenase & 1.8 & gi|70986839|ref|XP_748907.1| \\
\hline KMK61241.1 & catalase & 3.94 & gi|846915457|gb|KMK61241.1| \\
\hline EAL89500.1 & ubiquinone biosynthesis monooxgenase (COQ6) & 2.23 & gi|70993382|ref|XP_751538.1| \\
\hline EAL90759.1 & catechol dioxygenase & 1.8 & gi|70996084|ref|XP_752797.1| \\
\hline
\end{tabular}


b-3 VS b-2, c-2 VS c-1, c-3 VS c-1 and c-3 VS c-2 were set as comparison groups. Fold change $>1.2$ and $\mathrm{Q}$-value $<$ 0.05 were used to screen the significant difference proteins in single experiment. For repeated experimental data, the final differential protein was screened by fold change $>$ 1.2 (average of all comparison group ratios) and P-value $<0.05$ ( $\mathrm{t}$-test of all comparison groups). Figure 2 shows the number of differential proteins between comparison groups. The number of differential proteins between parallel samples was significantly smaller than that between B-1 and C-11. Comparing the secretion of A. fumigatus G-13 protein from B-1 (static culture with p-coumaric acid and microcrystalline cellulose as carbon source) and C-11 (shake flask culture with sinapic acid, carboxymethyl cellulose and glucose as carbon sources) samples, there are a total of 3862 protein spots were found to change significantly, with 2103 proteins down-regulated and 1759 proteins up-regulated.

\subsection{Differential protein biological information analysis}

Table S4 shows information on the 50 major proteins that have been significantly up-regulated, and Table S5 shows information on the 50 major proteins that have been significantly down-regulation. As can be seen from Tables S4 and Table S5, the proteins which are up-regulated are mainly proteins related to telomere and ribosome synthesis related proteins, monosaccharide transport proteins, chromosomal synthesis proteins, and glycohydrolysis proteins. The downregulated proteins are mainly endonuclease/exonuclease/ phosphatase family proteins, integral membrane transport proteins, and metal ion transport proteins.

The degradation of lignin by fungi is generally in the presence of $\mathrm{O}_{2}$, glucose oxidase can oxidize the corresponding substrates to produce $\mathrm{H}_{2} \mathrm{O}_{2}$. In the presence of $\mathrm{H}_{2} \mathrm{O}_{2}$, manganese peroxidase, lignin peroxidase and laccase synergistically act on the ether bond and ester bond linking the lignin structural units. After the lignin is decomposed into structural units, the $\mathrm{C}_{\alpha}-\mathrm{C}_{\beta}$ bond between cinnamyl alcohol is broken into two molecules of cinnamyl alcohol, and the cinnamyl alcohol is broken into small molecular compounds to complete the degradation of lignin. Therefore, enzymes involved in the lignin degradation process mainly include three types: $\mathrm{H}_{2} \mathrm{O}_{2}$ production enzyme system (eg, glucose oxidase, pyranose oxidase, glyoxal oxidase, methanol oxidase and aromatic aldehyde oxidase), lignin oxidase system (including peroxidases and laccases two categories) and other enzyme systems [methylase, cellobiose/quinone reductase system and aromatic ring cracking enzyme system (including oxidoreductases and hydrolases, with particular reference to monooxygenase, dioxygenase and amine oxidase)]. See Table 2 for information on differential proteins associated with lignin degradation. According to Table 2, C-11 compared with B-1 the differential protein of lignin degradation that is significantly up-regulated may include: catalase, ubiquinone biosynthesis monooxygenase (COQ6), FDA monooxygenase, catechol dioxygenase, quinone oxidoreductase, protoporphyrinogen oxidase, peroxisome 3-ketoacyl-CoA thiolase (KAT1), galactose oxidase, aldehyde ketone reductase (AKR), plant acyl-CoA dioxygenase family protein, etc.

The functions and metabolic pathways of these differentially expressed proteins were analyzed by GO database and KEGG database, and 607 GO entries were obtained. Table S6 shows the GO results of significant enrichment of differentially expressed proteins. Significantly enriched GO entries are mainly: proton-transporting V-type ATPase complex $(\mathrm{P}$ value $=0.1380685)$, peroxisome $(\mathrm{p}$-value $=0.1795317)$, proton-transporting ATP synthase complex $(\mathrm{p}$-value $=0.224264)$, protein-DNA complex $(\mathrm{p}$-value $=0.2853196)$, proteasome complex $(\mathrm{p}$ value $=0.2967193)$, preribosome $(\mathrm{p}$-value $=0.4544336)$. The main enrichment items of lignin-related differential proteins are located in mitochondrial oxoglutarate dehydrogenase complex, oxoglutarate dehydrogenase complex, peroxisome and its enzymatic membrane, matrix and other related items. The major enrichment entries for lignin-related differential proteins are located in mitochondrial oxoglutarate dehydrogenase complex (p-value $=0.00063487$ ), oxoglutarate dehydrogenase complex (p-value $=0.000478396)$, peroxisomal

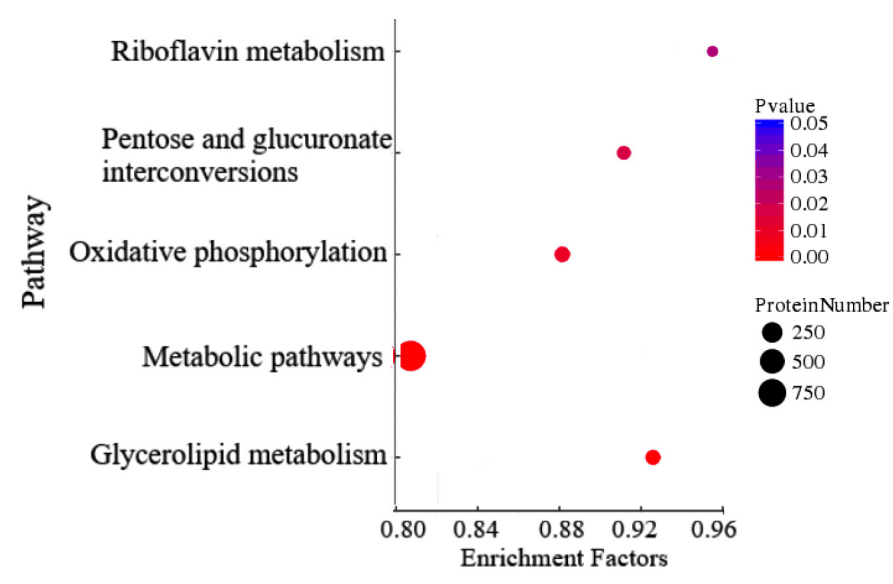

Figure 3. Schematic diagram of pathway enrichment of differential abundance protein 
$(\mathrm{p}$-value $=0.001235362)$, peroxisomal membrane $(\mathrm{p}-$ value $=0.000875123)$ and peroxisomal matrix ( $\mathrm{p}$-value $=0.000377498$ ) ect.

To further investigate the function of these proteins, the differential abundance proteins were mapped to 128 metabolic pathways by KEGG database alignment. Figure 3 is a schematic diagram of major enrichment, and some key pathways are shown in Table S7. Among them, metabolic pathway (p-value $=0.00087713$ ) is the most representative. Other significant enrichment pathways include: ribosome ( $\mathrm{p}$-value $=0.3112128 \mathrm{ko03010})$, glycerolipid metabolism ( $\mathrm{p}$-value $=0.000988395$ ko55061), oxidative phosphorylation ( $\mathrm{p}$-value $=0.01003147 \mathrm{ko00190}$ ), pentose and glucuronate interconversions ( $\mathrm{p}$-value $=0.01563957$ ko00040), riboflavin metabolism $(\mathrm{p}$-value $=0.02723311$ Ko00740), purine metabolism ( $\mathrm{p}$-value $=0.3461633$ ko0023), pyrimidine metabolism ( $\mathrm{p}$-value $=0.6759848$ ko00240), protein processing in endoplasmic reticulum $(\mathrm{p}$-value $=0.6816811 \mathrm{ko04141})$, glycine, serine and threonine metabolism $(\mathrm{P}=0.7686209$ ko00260).

\section{Discussion}

\subsection{Lignin degradation-related protein}

\subsubsection{Ferulic acid esterase and related proteins}

Ferulic acid esterase (EC 3.1.1.73, FAE) is a subclass of carboxylesterases and belong to the hydrolase family (Pinto, 2015; Takuya et al., 2009). FEA can hydrolyze ester bonds in methyl ferulate, oligosaccharide ferulic acid esters and polysaccharide ferulic acid esters. It can cut off the crosslinking between polysaccharide-polysaccharide and polysaccharide- lignin in cell wall, which is beneficial to the degradation of polysaccharides and release of lignin in cell wall materials. Ferulic acid esterase can synergize with xylanase and cellulase to increase the release of ferulic acid and reducing sugar, and it can also react synergistically with other enzymes to produce ferulic acid and carbohydrates (Yang et al., 2009). In this study, ferulic acid esterase expression was observed in both experimental groups, and there were significant differences in expression. That is, ferulic acid esterase is an enzyme that is induced when medium contains lignocellulosic substances (Chi et al., 2017). For example, Schizophyllum commune can produce highly active ferulic acid esterase in a medium that uses cellulose as a carbon source, and when a substrate with high xylan content (such as xylan and wheat bran) is used as a carbon source, it is hardly produced (Li et al., 2016). In this study, A. fumigatus G-13 induced the production of ferulic acid esterase in a medium containing lignin (ie, p-coumaric acid and sinapic acid), and its expression varies significantly due to the differences in composition of medium.

\subsubsection{Peroxisome-related proteins}

In the process of fungal degradation of lignin, peroxidase acts on the ether and ester bonds connecting the lignin structural units, so that the lignin is decomposed into a single structural unit. Afterwards, the cellobiose/quinone reductase system and the aromatic ring cracking enzyme system act synergistically, to further break the lignocellulose into small molecules and promote complete degradation of lignin (Yu et al., 2003). In the degradation pathway of lignin peroxidase, the following proteases were significantly down regulated: fructose amine oxidoreductase, isoamyl alcohol oxidase, ferulic acid ester enzyme B-1, ferulic acid ester enzyme B-2, peroxidase and peroxisome oxidase (Pan et al., 2011), 1,4-(1,3,1,4)- $\beta$-D-glucan 4-glucanohydrolase, cellobiose dehydrogenase, endoglucanase $\mathrm{D}$, carboxymethyl cellulase, avicelase, alkali cellulose and cellulase A3 (Hernández-Ramírez et al., 2018). Cellobiose dehydrogenase can break hydrogen bonds through a single electron oxidation pathway, resulting in the de-chaining and depolymerization of the fiber supramolecular structure, forming short fibers. Therefore, it has the ability to generate hydroxyl radicals and reduce various free radicals and degrade cellulose. Its down-regulation also indicates that under $\mathrm{C}-11$ conditions, the ability of A. fumigatus G-13 to degrade cellulose is enhanced. The possible cause is the presence of glucose in substrate under $\mathrm{C}-11$ conditions, glucose is a rapid utilization substrate, sinapic acid and carboxymethyl cellulose are slow-utilizing substrates. Strain uses glucose to grow and metabolize, and their metabolites inhibit the production of enzymes, that is, metabolites inhibit the process of glucose decomposition (An et al., 2015).

\subsubsection{Cellobiose/quinone reductase system and aromatic ring cracking enzyme system}

Cytochrome P450 monooxygenase, amine oxidase, flavin monooxygenase, $\beta$-glucuronide hydrolase, urate-1,2-dioxygenase, cellobiose dehydrogenase ect can be used for cellobiose/quinone reduction or aromatic ring cracking (Wang et al., 2014). Among them, cytochrome P450 (CYP) is a superfamily protein containing heme as a cofactor and it is a heme protein. CYPs utilize various lignocellulosic macromolecules and corresponding degradation intermediates as substrates in the enzymatic reaction. In general, they are terminal oxidases in electron transfer chains (Robb et al., 2018). The most common reaction catalyzed by CYP is a monooxygenase reaction. One oxygen atom is inserted into the aliphatic site of organic substrate and the other oxygen atom is reduced to water. Therefore, the decrease in CYP expression indicates that $\mathrm{C}-11$ is more efficient than B-1 in the third stage of lignin degradation. Flavin-binding monooxygenase works similarly to CPY. It can act on copper and iron and cytochrome $\mathrm{C}$, but its role needs to be localized in the mitochondria.

\subsection{Enrichment analysis of other differential proteins}

\subsubsection{Carbon source and energy metabolism}

In the metabolism of carbon sources, the most important way is glycolysis. Under C-11 conditions, A. fumigatus G-13 relies almost exclusively on the glycolysis pathway 
to provide energy supply. In this study, the proteins associated with glycolysis were mainly phosphoglycerate coolase dehydrogenase and ethanol dehydrogenase, and the expression of these two enzymes was up-regulated in C-11 compared to B-1. It indicated that in the presence of glucose, this strain used glucose fermentation for energy metabolism to achieve cell proliferation.

The pentose phosphate pathway was also a major metabolic pathway. The proteins associated with the pentose phosphate pathway screened in this study were mainly 6-phosphate glucose dehydrogenase and 6-phosphoric acid decarboxylase. In the pentose phosphate pathway, 6-phosphate glucose dehydrogenase catalyzed the production of glucose 6-phosphate to phosphorgluconic acid and NADPH. 6-phosphate decarboxylase catalyzed the production of D-nucleoside sugar-phosphoric acid by glucose 6-phosphate, and simultaneously produced NADPH, which further formed ribose-5-phosphate. NADPH generated in this pathway can be used to reduce other substances and participate in the synthesis of reducing biomolecules. The intermediate product D-nucleoside sugar-phosphoric acid is one of the main components of nucleic acids and their important coenzymes (Valdes et al., 2008). 6-phosphate glucose dehydrogenase and 6-phosphoric acid decarboxylase are rate-limiting enzymes. They are more suitable for characterizing the redox state of mycelium grown in the C-11 environment due to their special signaling effects. In this study, the expression of these two enzymes (6-phosphate glucose dehydrogenase and 6-phosphoric acid decarboxylase) was up-regulated under B-1 conditions. It indicated that the mycelium initiate multiple reactions to coordinate and cooperate with each other to carry out metabolic activities. Meanwhile they also need to generate more energy to maintain their own cell growth and reproduction.

\subsubsection{Glyceride metabolism related proteins}

In the biological metabolism of glyceride, acyltransferase is an extremely important precursor, and acyltransferase is catalyzed by carboxylase. The reaction is a rate-limiting process and the carboxylase is a variable-structure enzyme. Under catalytic conditions, the activity of carboxylase will be enhanced, the yield of corresponding acyltransferase will be increased, and catalytic ability of the substrate will be increased, thereby producing more target products. However, the increase of long chain will inhibit the activity of carboxylase and reduce the production of corresponding enzymes, so that the content of glyceride will also decrease. Carboxylase activity is also affected by phosphorylation of cyclic adenosine phosphate (cAMP) phosphorylation. During the phosphorylation of carboxylase, the enzyme activity is inhibited, the content of corresponding enzyme is relatively reduced, and the catalytic ability of carboxylase to target products is weakened. After phosphorylation, the inhibition of enzyme disappears, the initial activity is restored, the production of corresponding enzyme is increased, and the catalytic capacity of substrate is also increased (Long et al., 2018).
Carrier protein polymers (FabZ2, FabG2, FabD and FabH) play a leading role in catalyzing the metabolism of glycerides. These multifunctional enzymes catalyze the metabolism of glycerides, and the energy required in the catalytic process (including ATP and NADPH) is produced by the pentose phosphate pathway in sugar metabolism or related pathways. In this study, a large number of glyceride metabolism-related proteins in C-11 changed significantly, and their expressions were up-regulated. This indicates that the changes of lignin model compounds and the addition of glucose promote the enhancement of the secretory enzyme activity of the mycelium. That is, C-11 is more conducive to the growth of A. fumigatus G-13.

\subsubsection{Nucleotide metabolism}

Among the nucleotide metabolism-related proteins, the proteins that have undergone significant changes were mainly purine biosynthesis and metabolism-related proteins, whose main function is to catalyze the production of guanylic acid (GMP) to inosine acid (IMP). Bifunctional purine biosynthetic protein (PurH) is the last two steps in biosynthesis of deoxyribonucleotides. First, PurH catalyzes the 5-aminoimidazole-4-carboxamide$1-\beta$-D- ribofuranoside (AICAR) to 5-formamidoimidazole-4-carboxamide ribotide (FAICAR), and then cyclic hydrolase continues to catalyze FAICAR to produce inosine. Adenylate succinate synthetase (PurA) catalyzes the formation of adenylate succinic acid (SAMP) by adenylate, and adenylate succinate lyase (PurB) makes adenosine succinate to produce adenosine monophosphate (AMP). Adenylate kinase catalyzes AMP to produce adenosine diphosphate, which further generates adenosine triphosphate (ATP). The decrease in PurA content can increase concentration of hypoxanthine in nucleotide metabolism, and then synthesizing RNA and DNA (Zhang et al., 2008).

In this study, compared with B-11 and C-11, various nucleotide metabolism-related proteins were observed to change significantly, and their expressions were downregulated. It indicated that B-1 inhibited the growth of the cells, leading to a decrease in the activity of related enzymes in the metabolism of deoxyribonucleotides during cell growth and reproduction, and the synthesis of related substances is reduced. It also enabled the metabolic intermediates to synthesize as many ribonucleotides and deoxyribonucleotides as possible. The results are similar to previous analyses of fungal proteomics under different cellulosic substrates.

\subsubsection{Mutual transformation of pentose and glucuronic acid}

The pentose phosphate pathway is carried out in cytoplasm and provides ribose 5-phosphate for the biosynthesis of nucleotides and nucleic acids. It also provides NADPH for various metabolic reactions and maintains the reduced state of glutathione. The physiological significance of uronic acid pathway is production of active 
glucuronic acid (UDP glucuronic acid), which is an important binder in biotransformation. It can combine with many metabolites and is also a donor of glucuronic acid. Figure S4 shows the schematic diagram of mutual conversion of pentose and glucuronic acid. Under this pathway: the synthesis of 2-dehydor-3-deoxy-D-gluconate and (4S)-4,6-dihydroxy-2,5- dioxohexanoate is reduced, and at the same time, poly $(1,4)-\alpha-D$-polygalacturonic acid, digalactose, $\mathrm{D}$-galacturonate and other synthesis are also reduced. In this study, glucose 6-phosphate dehydrogenase (G6P) and poly-galacturonide glycanohydrase (PG) were significantly down-regulated. Because PG can degrade pectin and damage cell walls, the presence of PG can assist strains to degrade lignin in the biodegradation of natural lignin. Compared with B-1 and C-11, the key proteins (G6P, PG) in the process of mutual conversion of pentose and glucuronic acid were significantly downregulated. It is indicated that under B-1 conditions, the energy metabolism of mycelium was significantly affected, and the synthesis of enzymes related to the degradation of lignin substrates was also reduced. In general, the changes of ligin model compounds and the addition of glucose can enhance the energy metabolism of the cells and assist the degradation of lignin by bacteria.

\subsubsection{Monoammine metabolism related proteins}

Monocyclic $\beta$-lactam is a $\beta$-lactam compound. Compared with most $\beta$-lactams, the $\beta$-lactam ring is isolated and not closed (Taherzadeh \& Karimi, 2008). It has antibiotic effects on some aerobic Gram-negative bacteria (such as Neisseria and Pseudomonas). Under the condition of B-1, a number of proteins related to metabolism of monoamines were found, and their expression was significantly up-regulated. Its structure is simpler than penicillin and cephalosporin, and its chemical properties are more stable than those of carbapenems (Stringer et al., 2015). It can be speculated that under B-1 conditions, A. fumigatus G-13 can synthesize a large amount of monoamine to inhibit other microbial growth. Under these conditions, the secreted bacteriostatic substances have the potential to be used as antibiotics and used in clinical research and disease treatment.

\section{Conclusions}

The secretome data showed that A. fumigatus G-13 has a complex enzymatic system implicated in lignocellulose degradation. Differential proteins are relatively concentrated in energy metabolism pathways. Synthesis of Dnucleoside sugar-phosphoric and NADPH through the pentose phosphate pathway, promotes the metabolism of nucleotides to generate enough energy to maintain the proliferation of cells, and assists A. fumigatus G-13 to produce a large number of lignin-degrading enzymes. Among them, the key enzyme ferulic acid esterase can destroy the cross-linking of polysaccharide-lignin and act on the carboxylate bond. Simultaneously it can cooperate with a series of lignin degrading enzymes to further break the lignocellulose into small molecules. This study discussed the mechanism of lignin 14 biodegradation, which will contribute to the more systematic regulation of lignin biodegradation in the future.

\section{Acknowledgements}

The authors would like to thank the National Natural Science Foundation of China [No: 21776054] supporting this research.

\section{References}

Adav, S. S., Ravindran, A., \& Sze, S. K. (2015). Quantitative proteomic study of Aspergillus Fumigatus secretome revealed deamidation of secretory enzymes. Journal of Proteomics, 119, 154-168. https://doi.org/10.1016/j.jprot.2015.02.007

An, Q., Wu, X. J., Wu, B., \& Dai, Y. C. (2015). Effects of carbon and nitrogen sources on lignocellulose decomposition enzyme activities in Flammulina velutipes. Mycosystema, 34, 761-771.

Arulmani, M., Sunili, S. A., \& Siu, K. S. (2011). iTRAQ-based quantitative secretome analysis of Phanerochaete chrysosporium. Journal of Proteomics, 75(2), 642-654.

https://doi.org/10.1016/j.jprot.2011.09.001

Chi, D. H., Giap, V. D., Anh, L. P. H., \& Nghi, D. H. (2017). Feruloyl esterase from Alternaria tenuissima that hydrolyses lignocellulosic material to release hydroxycinnamic acids. Applied Biochemistry and Microbiology, 53(6), 654-660. https://doi.org/10.1134/S0003683817060047

Dawoud, M. E. A., \& Abu-Taleb, A. M. (2012). Evaluation of the roles of physical (osmotic, gamma irradiation and/or heat shock) stress factors on enzyme activities and protein accumulation in Pleurotus ostreatus mushroom and its descendent progenies. Journal of Food Agriculture \& Environment, 10(2), 22-32.

Grabber, J. H. (2005). How do lignin composition, structure, and cross-linking affect degradability? A review of cell wall model studies. Crop Science, 45(3), 820-831.

https://doi.org/10.2135/cropsci2004.0191

Hernández-Ramírez, L. C., Trivellin, G., \& Stratakis, C. A. (2018). Cyclic 3', 5'-adenosine monophosphate (cAMP) signaling in the anterior pituitary gland in health and disease. Molecular and Cellular Endocrinology, 463, 72-86. https://doi.org/10.1016/j.mce.2017.08.006

Himmel, M. E., Ding, S. Y., Johnson, D. K., Adney, W. S., Nimlos, M. R., Brady, J. W., \& Foust, T. D. (2007). Biomass recalcitrance: Engineering plants and enzymes for biofuels production. Science, 315(5813), 804-807. https://doi.org/10.1126/science.1137016

Ji, X. L., Zhang, W. T., Gai, Y. P., Lu, B. Y., Yuan, C. Z., Liu, Q. X., \& Mu, Z. M. (2012). Patterns of lignocellulose degradation and secretome analysis of Trametes trogii MT. International Biodeterioration and Biodegradation, 75, 55-62. https://doi.org/10.1016/j.ibiod.2012.09.001

Li, K., Xu, F., \& Eriksson, K. E. (1999). Comparison of fungal laccases and redox mediators in oxidation of a nonphenolic lignin model compound. Applied and Environmental Microbiology, 65(6), 2654-2660. 
Li, W., Sun, Q. D., \& Wang, H. X. (2016). A review on research and applications of feruloyl esterase. Journal of Shandong Agricultural University (Natural Science Edition), 47, 628-635. https://doi.org/10.3969/j.issn.1000-2324.2016.04.029

Long, T. M., Tonsing-Carter, E. Y., Chan, W. C., Griend, D. V., Conzen, S. D., \& Szmulewitz, R. Z. (2018). Glucocorticoid receptor (GR)-mediated activation of cyclic-adenosine monophosphate (cAMP) pathway gene expression following androgen receptor $(\mathrm{AR})$ antagonism of prostate cancer. Cancer Research, 78(13), 944. https://doi.org/10.1158/1538-7445.AM2018-944

MacDonald, J., Suzuki, H., \& Master, E. R. (2012). Expression and regulation of genes encoding lignocellulose-degrading activity in the genus Phanerochaete. Applied Microbiology and Biotechnology, 94, 339-351. https://doi.org/10.1007/s00253-012-3937-Z

Pan, M. F., Jiang, M., \& Zhou, Z. W. (2011). Latest research advances in biodegradation of lignin. Materials Review, 25, 372-377.

Pinto, C. (2015). Feruloyl esterase: A principal biodegradative enzyme. In Bioprospects of Coastal Eubacteria (pp. 209-224). Springer, Cham. https://doi.org/10.1007/978-3-319-12910-5_12

Ray, A., Saykhedkar, S., Ayoubi-Canaan, P., Hartson, S. D., Prade, R., \& Mort, A. J. (2012). Phanerochaete chrysosporium produces a diverse array of extracellular enzymes when grown on sorghum. Applied Microbiology and Biotechnology, 93(5), 2075-2089. https://doi.org/10.1007/s00253-012-3907-5

Robb, C. S., Reisky, L., Bornscheuer, U., \& Hehemann, J. (2018). Specificity and mechanism of carbohydrate demethylation by cytochrome P450 monooxygenases. Biochemical Journal, 475(23), 3875-3886. https://doi.org/10.1042/BCJ20180762

Stringer, T. P., Guerrieri, D., Vivar, C., \& Praag, H. V. (2015). Plant-derived flavanol (-)epicatechin mitigates anxiety in association with elevated hippocampal monoamine and BDNF levels, but does not influence pattern separation in mice. Translational Psychiatry, 5, e493. https://doi.org/10.1038/tp.2014.135

Taherzadeh, M. J., \& Karimi, K. (2008). Pretreatment of lignocellulosic wastes to improve ethanol and biogas production: A Review. International Journal of Monoamine Sciences, 9(9), 1621-1651. https://doi.org/10.3390/ijms9091621
Takuya, K., Shinya, F., Hitoshi, S., \& Michio, K. (2009). Occurrence, properties, and applications of feruloyl esterases. Applied Microbiology and Biotechnology, 84(5), 803-810. https://doi.org/10.1007/s00253-009-2148-8

Tao, Y. Z., \& Guan, Y. T. (2003). Study of chemical composition of lignin and its application. Journal of Cellulose Science and Technology, 11, 42-50. http://en.cnki.com.cn/Article_en/ CJFDTOTAL-XWSK200301008.htm

Valdes, J., Pedroso, I., Quatrini, R., Dodson, R. J., Tettelin, H., Blake, R., Eisen, J. A., \& Holmes, D. S. (2008). Acidithiobacillus ferrooxidans metabolism: From genome sequence to industrial applications. BMC Genomics, 9, 597. https://doi.org/10.1186/1471-2164-9-597

Wang, S. Y., Jiang, S. C., Wang, K. Y., Wang, Y., \& Zhang, M. P. (2014). Research process of cytochrome P450 in plant. Jilin Vegetables, 4, 41-45. http://en.cnki.com.cn/Article_en/CJFDTotal-JLSS201404031.htm

Wilkins, M. R., Sanchez, J. C., Gooley, A. A., Apple, R. D., Humphery-Smith, I., Hochstrasser, D. F., \& Williams, K. L. (1996). Progress with proteome projects why all proteins expressed by a genome should be identified and how to do it. Biotechnology and Genetic Engineering Review, 13(1), 19-50. https://doi.org/10.1080/02648725.1996.10647923

Yang, H. J., Yue, Q., Cao, Y. C., Zhang, D. F., \& Wang, J. Q. (2009). Effects of crude feruloyl and acetyl esterase solutions of Neocallimastix sp. YQ1 and Anaeromyces sp. YQ3 isolated from Holstein steers on hydrolysis of Chinese wildrye grass hay, wheat bran, maizebran, wheat straw and corn stalks. Animal Feed Science and Technology, 154(3-4), 218-227. https://doi.org/10.1016/j.anifeedsci.2009.09.006

Yu, H. Y., Zeng, G. M., Hu, T. J., \& Chen, Y. N. (2003). Advance in biodegradation of lignin by fungus and expectation of research in aerobic compost. Journal of Chinese Biotechnology, 23(10), 57-61.

Zhang, Y., Morar, M., \& Ealick, S. E. (2008). Structural biology of the purine biosynthetic pathway. Cellular and Molecular Life Science, 65(23), 3699-3724. https://doi.org/10.1007/s00018-008-8295-8 